\title{
Resultados del tratamiento con revascularización distal y ligadura intermedia, de la isquemia de mano después de acceso vascular para hemodiálisis*
}

\author{
Drs. REGINALD ROJAS N. ${ }^{1}$, ANDRÉS ROJAS G. ${ }^{1}$, RODRIGO MARTÍNEZ H. ${ }^{1}$ \\ 1 Servicio de Cirugía Hospital de Puerto Montt, Puerto Montt. \\ Chile.
}

\begin{abstract}
Distal revascularization-interval ligation for ischemic steal syndrome after hemodialysis access. Experience in 23 patients

Background: The creation of vascular accesses for hemodialysis can cause distal ischemia and steal syndromes. Aim: To assess the effectiveness of the technique of distal revascularization-interval ligation to alleviate distal ischemia and preserve vascular access. Material and Methods: Retrospective review of medical records, identifying 23 patients (13 women, aged between 24 and 79 years), with distal ischemia secondary to a vascular access for hemodialysis, that were treated with distal revascularization-interval ligation. Patient characteristics and outcome of the surgical procedure were recorded. Results: Fourteen patients were diabetic and 15 had high blood pressure. All had the vascular access in the elbow, 20 were done with vein and three were prosthetic. Steal appeared in a lapse ranging from hours to six years after performing the procedure. In 14 patients it appeared before 12 months. Revascularization was performed between 1 day and three months after the appearance of the steal syndrome. Seventeen patients (74\%) had a substantial relief of ischemic symptoms, with healing of ulcers and digital amputations. Three patients died soon after the procedure (13\%). In two the pain persisted, requiring a banding of the access, that finally became thrombosed. One patient required a distal forearm amputation. Thirteen patients (56\%) had a late death after the procedure. After revascularization, the vascular accesses were used for a mean of two years. Conclusions: Revascularizationinterval ligation relieves distal ischemia and maintains the patency of the vascular access for hemodialysis. Patients with steal syndrome secondary to vascular access are of high risk.
\end{abstract}

Key words: Steal syndrome, hemodialysis, vascular access.

\section{Resumen}

Objetivo: Investigar la efectividad de la técnica de revascularización distal y ligadura intermedia (RDLI) en aliviar la isquemia y preservar el acceso arteriovenoso. Material y Métodos: Una revisión retrospectiva de fichas clínicas (1990-2010), identificó 26 pacientes con robo. Se excluyen 3, que se sometieron a ligadura del acceso; 23 fueron tratados con RDLI. Se obtuvo información demográfica, comorbilidades,

*Recibido el 3 de agosto de 2011 y aceptado para publicación el 19 de diciembre de 2011.

Correspondencia: Dr. Reginald Rojas N.

Padre Harter 577, Puerto Montt, Chile.

reginaldrojas@gmail.com 
antecedentes del acceso, morbimortalidad y respuesta al tratamiento. Resultados: De los 23 pacientes, 13 eran mujeres y 10 hombres, con edad promedio de 59 años (24-79 años). Catorce (61\%) eran diabéticos y quince (65\%) hipertensos. Todos con acceso en pliegue del codo, 20 con vena y 3 protésicos. La latencia en aparición del robo ocurrió antes de 12 meses, en 14 pacientes (78\%); con rango desde horas a 6,5 años. La revascularización se realizó en tiempo variable entre 1 día y 13 meses; sólo 8 pacientes antes de los 30 días. El 74\% de los pacientes (17), tuvo mejoría sustancial o completa de manifestaciones isquémicas, cicatrizando úlceras y amputaciones digitales. Tres fallecieron precozmente; en 2 pacientes, persistió el dolor, requiriendo banding del acceso, que finalmente se trombosó; un paciente debió ser amputado del antebrazo distal, pese a revascularización, al no ceder cuadro infeccioso. La mortalidad operatoria fue de 13\%, correspondiendo a 3 pacientes muy deteriorados con isquemia severa. La mortalidad tardía fue de 56,5\% (13 pacientes). El tiempo promedio de uso del acceso fue de 2 años, post revascularización. Conclusión: La RDLI es el procedimiento de elección en el tratamiento del robo, ya que elimina efectivamente la isquemia y mantiene en uso el acceso. Los pacientes con robo, especialmente diabéticos, representan un grupo de alto riesgo y mortalidad.

Palabras clave: Robo, acceso vascular, revascularización distal.

\section{Introducción}

La creación de un acceso vascular para hemodiálisis, provoca cambios en la irrigación de la extremidad, que pueden derivar en isquemia distal o robo. Este fenómeno puede manifestarse con frialdad de dedos, parestesias, dolor, úlceras y necrosis, requiriendo tratamiento alrededor del $4 \%$ de los pacientes con accesos autólogos o protésicos.

No se dispone de métodos preoperatorios confiables para predecir esta complicación, aunque tienen mayor riesgo los pacientes diabéticos y la presencia de accesos en pliegue del codo o proximales.

Existen varios procedimientos quirúrgicos para corregir la isquemia, con diferentes resultados. Actualmente, la revascularización distal y ligadura intermedia (RDLI), descrita por Schanzer ${ }^{1}$ en 1988, es considerada la estrategia más eficaz en resolver la isquemia y mantener el acceso permeable, especialmente en pacientes con fístula arteriovenosa funcionante y disponibilidad de lecho vascular limitada.

El objetivo de este trabajo, fue investigar la efectividad de la técnica de revascularización distal y ligadura intermedia, en aliviar la isquemia y preservar el acceso arteriovenoso.

\section{Material y Método}

Entre mayo de 1990 y junio de 2010, se han efectuado en nuestro hospital 1.516 accesos vasculares autólogos y protésicos, incluyendo reparaciones y otros procedimientos relacionados. En este período, se identificaron 26 pacientes (1,71\%) con isquemia severa de la mano, secundarias a su acceso vascular. Tres fueron sometidos sólo a ligadura del acceso, por isquemia avanzada de mano. Veintitres fueron sometidos a revascularización distal y ligadura intermedia (RDLI); estos últimos constituyen el objeto del presente estudio.
Se obtuvo información de estos pacientes, mediante la revisión retrospectiva de las fichas clínicas, registro civil, contacto telefónico con pacientes y/o familiares, y centro de diálisis.

Se analizan comorbilidades, sexo, edad, manifestaciones clínicas de isquemia, antecedentes del acceso vascular, que precedió a la presentación del robo, antecedentes del procedimiento de revascularización, latencia en aparición del robo, diagnóstico, complicaciones y morbimortalidad operatoria y tardía.

La indicación de revascularización, se determinó por la severidad de la isquemia: presencia de déficit neurológico sensitivo o motor, dolor de reposo y/o presencia de úlcera isquémica o necrosis.

No existió una pauta uniforme de evaluación para el diagnóstico de la isquemia, pero estuvo fundamentalmente basada en los hallazgos clínicos en la mayoría de los pacientes, dada la severidad de manifestaciones al ingreso. Por carencia de laboratorio vascular en nuestra institución, se utilizó menos frecuentemente pletismografía con medición de volumen de pulso y presiones segmentarias, y fotopletismografía digital con y sin oclusión de la fístula. El ecodoppler de vasos arteriales de la extremidad superior para determinar tamaño del ostium y dirección del flujo, fue utilizado ocasionalmente, por disponibilidad limitada. No se realizó arteriografía ni flebografía en ningún paciente.

La técnica quirúrgica usada, consistió en la ligadura arterial inmediatamente distal al acceso y revascularización mediante la confección de puente venoso o protésico, cuya anastomosis en la arteria donante fue hecha 5-10 cm proximal al ostium de la fístula.

La respuesta al tratamiento, se determinó por la desaparición o mejoría clínica de la isquemia, y necesidad de amputación.

La evaluación postoperatoria de función del acceso, se determinó por presencia de soplo y frémito, 
asociado a la mantención de hemodiálisis por la fístula. La permeabilidad del puente se estimó por palpación de pulso distal, confirmado con la presencia de flujo pulsátil, con doppler, sobre él y las arterias distales.

\section{Resultados}

Todos los pacientes con robo, sometidos a la revascularización con la técnica RDLI, fueron intervenidos en nuestro hospital, centro de referencia del área geográfica cubierta por el Servicio de Salud Llanquihue, Chiloé y Palena.

La edad de los pacientes, osciló entre los 24 y 79 años, con un promedio de 59,4 años.

Diez eran hombres y 13 de sexo femenino.

De los 23 pacientes, 15 (65\%) eran hipertensos, 14 (61\%) diabéticos, 4 (17\%) portadores de enfermedad arterial oclusiva de extremidades inferiores, 3 (13\%) con cardiopatía coronaria y 1 (4,3\%) con lupus eritematoso generalizado.

Todos los pacientes tenían acceso en el pliegue del codo; 20 con vena y 3 con prótesis (PTFE) (Tabla 1).

Las manifestaciones de isquemia se presentaron antes de los 12 meses en 14 pacientes, en 4 de ellos luego de escasas horas de construido el acceso (uno protésico, axilohumeral). Un segundo acceso protésico presentó manifestaciones isquémicas 6 días después. En el resto de los pacientes la latencia en la aparición de isquemia ocurrió entre 12 a 24 meses, incluyendo un paciente con antecedente de 3 accesos autólogos en la misma extremidad, y que se evidenció tardíamente a los 6,5 años (Tabla 2).

Catorce pacientes tenían antecedentes de múltiples accesos previos construidos (2, 3 y 5), al momento de la aparición de la isquemia.

La forma de presentación de los síntomas y signos isquémicos fue diversa entre los pacientes: frialdad y cianosis (1), parestesias (2), dolor de reposo (12), úlcera isquémica (6), necrosis seca de dedos (1), flegmón necrotizante dedos y dorso de mano (1).

La confirmación del diagnóstico, estuvo basada en los hallazgos clínicos en los 23 pacientes; se complementó con mediciones de volúmenes de pulso, presiones segmentarias y fotopletismografía digital en 9 y con doppler de arterias radiales y cubitales en 3 .

Los pacientes fueron sometidos a la técnica RDLI, entre 1 día y 13 meses luego de haberse presentada la isquemia. Sólo ocho fueron tratados antes de los 30 días.

Se usó para la revascularización vena safena interna (18 pacientes), vena cefálica (1 paciente) y conducto protésico (PTFE) en 4 pacientes.
La distancia entre la anastomosis proximal del puente y el ostium del acceso fue variable: $10 \mathrm{~cm}$ (13 pacientes), $8 \mathrm{~cm}$ (1 paciente), $7 \mathrm{~cm}$ (2 pacientes), $6 \mathrm{~cm}$ (1 paciente) y $5 \mathrm{cms}$ (2 pacientes). Sin información en 4 pacientes.

La arteria donante fue la humeral en todos los pacientes, ligándose la arteria con polipropileno 4/0 y/o hemoclip, inmediatamente distal al acceso arteriovenoso y proximal a la anastomosis distal hecha a la arteria radial o humeral.

No se observó ninguna diferencia en la mejoría clínica de la perfusión distal de la extremidad, con los diferentes conductos ni distancias anteriormente señaladas (5 a 10 cm). Ningún puente se trombosó ni requirió ningún procedimiento adicional en el post operatorio hasta su alta.

Entre las complicaciones, destacaron infección de la herida operatoria (2 pacientes) por Staphylococcus aureus y Acinetobacter, hematoma del túnel subcutáneo (1 paciente), sangrado de herida operatoria (1 paciente), pérdida de líquido seroso (1 paciente); ninguno requirió reintervención.

Tabla 1. Acceso arterioso venoso previo a la aparición de robo $(n=23)$

\begin{tabular}{|lrr|}
\hline Acceso vascular & n & (\%) \\
\hline Acceso arterio venoso con vena: & & \\
$\quad$ Húmero cefálica & 18 & (78) \\
Húmero basílica & 1 & \\
Húmero cefálico basílica & 1 & \\
Acceso arterioso venoso protésico (PTFE): & & (13) \\
$\quad$ Axilo humeral & 3 & \\
\hline
\end{tabular}

Tabla 2. Latencia en presentación de robo $(n=19)$

\begin{tabular}{|lrr|}
\hline Tiempo & $\mathbf{n}$ & $\mathbf{( \% )}$ \\
\hline Inmediato (horas) & 4 & $(21)$ \\
4 - 7 días & 3 & $(16)$ \\
2 meses & 1 & $(5)$ \\
3 meses & 2 & $(11)$ \\
4 meses & 2 & $(11)$ \\
5 meses & 1 & $(5)$ \\
11 meses & 1 & $(5)$ \\
13 meses & 1 & $(5)$ \\
15 meses & 1 & $(5)$ \\
18 meses & 1 & $(5)$ \\
\hline 2 años & 1 & $(5)$ \\
6,5 años & 1 & $(5)$ \\
\hline
\end{tabular}


Tabla 3. Causas de mortalidad precoz y tardía en nefrópatas con robo arterial tratados con revascularización distal y ligadura intermedia

\begin{tabular}{|lc|}
\hline Causas de mortalidad & n pacientes \\
\hline Mortalidad global & $16(69,5 \%)$ \\
\hline Mortalidad operatoria (< 30 días) & $3(13 \%)$ \\
Infarto cerebral & 1 \\
Sepsis & 1 \\
Deterioro tóxico metabólico & 1 \\
\hline Mortalidad tardía (> 30 días) & $13(56,5 \%)$ \\
Enf. diverticular complicada (rectorragia) & 2 \\
Hematoma subdural agudo & 1 \\
Deterioro tóxico metabólico & 5 \\
Infarto miocardio & 1 \\
Sepsis +falla orgánica múltiple & 3 \\
Desconocido & 1 \\
\hline
\end{tabular}

Se observó mejoría clínica en la irrigación de la mano en 17 (74\%) pacientes tratados con la técnica RDLI, cediendo la frialdad y dolor rápidamente, y progresiva desaparición de las parestesias, con cicatrización de amputaciones distales de dedos mediano, meñique y del anular (3 pacientes). Sin embargo, tres pacientes fallecen precozmente no cicatrizando úlceras, pese a acceso y puente permeables. No cedió el dolor en 2 pacientes que requirieron banding a los 2,5 y 4,5 meses después, trombosándose tardíamente la fístula. Un paciente diabético, ciego y portador de enfermedad arterial oclusiva de extremidades inferiores, con gangrena isquémica de dedos y mano, fue amputado en el antebrazo, por persistencia de isquemia e infección. Al momento de la amputación el puente estaba permeable, y continuó usando el mismo acceso hasta su muerte, 4 años después.

La mortalidad operatoria, correspondió a tres (13\%) pacientes, los que fallecieron durante la hospitalización post revascularización a los 3, 4 y 12 días con puente y acceso funcionando, tratándose de individuos muy deteriorados por comorbilidades y enfermedad renal, que presentaron isquemia severa con dolor de reposo y úlcera isquémica.

Durante el período de observación, la mayoría de los fallecimientos tardíos (13 pacientes) ocurrió entre los 2-3 años post revascularización, con un rango entre 11 meses y 105 meses por diferentes causas (Tabla 3).

El seguimiento tardío de los pacientes tratados con RDLI, desde la presentación del primer caso (marzo 1996), al último (junio 2010), mostró que la mayoría (11) continuó usando el acceso hasta su fallecimiento o hasta la pérdida de éste por
Tabla 4. Tiempo de uso del acceso vascular en nefrópatas crónicos sometidos a RDLI (marzo de 1996 - junio de 2010)

\begin{tabular}{|c|c|c|}
\hline $\mathbf{P}$ & $\begin{array}{l}\text { Tiempo de uso de } \\
\text { acceso arteriovenoso }\end{array}$ & $\begin{array}{l}\text { Causa de término de diálisis } \\
\text { por acceso arteriovenoso }\end{array}$ \\
\hline 1 & 11 meses & Fallecido \\
\hline 2 & 105 meses & Fallecido \\
\hline 3 & 49 meses & Fallecido \\
\hline 4 & 4 meses & $\begin{array}{l}\text { Trombosis post banding. } \\
\text { Requirió otro acceso }\end{array}$ \\
\hline 5 & 67 meses & Fallecido \\
\hline 6 & 26 meses & Fallecido \\
\hline 7 & Desconocido & Fallecido \\
\hline 8 & 56 meses & $\begin{array}{l}\text { Trombosis. } \\
\text { Requirió otro acceso }\end{array}$ \\
\hline 9 & 16 meses & Fallecido \\
\hline 10 & 22 meses & $\begin{array}{l}\text { Ligadura acceso post sangrado. } \\
\text { Requirió otro acceso }\end{array}$ \\
\hline 11 & 23 meses & $\begin{array}{l}\text { Trombosis acceso. } \\
\text { Requirió otro acceso }\end{array}$ \\
\hline 12 & 7 meses & $\begin{array}{l}\text { Trombosis post banding. } \\
\text { Requirió otro acceso }\end{array}$ \\
\hline 13 & 12 días & Fallecido \\
\hline 14 & 9 meses & Fallecido \\
\hline 15 & 4 días & Fallecido \\
\hline 16 & 3 días & Fallecido \\
\hline 17 & 32 meses & $\begin{array}{l}\text { Trombosis acceso. } \\
\text { Requirió otro acceso }\end{array}$ \\
\hline 18 & 29 meses & En uso \\
\hline 19 & 23 meses & En uso \\
\hline 20 & 13 meses & En uso \\
\hline 21 & 9 meses & Fallecido \\
\hline 22 & Desconocido & Fallecido \\
\hline 23 & 48 meses & Fallecido \\
\hline
\end{tabular}

Promedio total del uso del acceso arteriovenoso excluyendo los tres pacientes fallecidos precozmente: 24,8 meses (rango 4 a 105 meses). P: paciente.

trombosis (5) y sangrado (1). Tres fallecieron precozmente antes de los 30 días, sin lograr usar el acceso. Actualmente, 3 pacientes, continúan en hemodiálisis a través de la fístula tratada con RDLI, 13, 23 y 29 meses después, sin recidiva de la isquemia y puente permeable. El tiempo de uso promedio del acceso, excluyendo a los fallecidos antes de los 30 días, fue de 24,8 meses (rango: 4 a 105 meses) (Tabla 4). 


\section{Discusión}

Aunque la incidencia reportada de robo isquémico varía entre $1,6 \%$ a $8 \%$, estudios prospectivos señalan que la incidencia actual de robo sintomático es mayor (15 a 20\%), probablemente por la incapacidad de los clínicos en reconocer esta complicación. En la actualidad, aproximadamente el $4 \%$ de los pacientes con acceso vascular requiere intervención quirúrgica por robo. En nuestra experiencia alcanzó al $1,7 \%$.

La presencia de una fístula arteriovenosa provoca una reversión del flujo sanguíneo en la arteria distal a la fístula, fenómeno que está presente en cerca del $80 \%$ de todos los accesos, sin embargo, no está siempre asociado a isquemia de la mano. La isquemia severa o robo de la mano ocurre cuando la irrigación colateral es insuficiente para mantener un flujo anterógrado adecuado a la mano; esto ocurre en presencia de enfermedad arterial oclusiva, vasculitis o fístula con alto débito.

La latencia en el inicio de los síntomas de robo, puede ser precoz o tardía. Los accesos protésicos, desarrollan con mayor frecuencia isquemia precoz en comparación a los realizados con vena, que lo hacen más tardíamente, en la medida que la vena se dilata. Según Lazarides et $\mathrm{al}^{2}$, el tiempo promedio de la aparición de síntomas es de 2 días en accesos protésicos y de 165 días en los autólogos. En la presente serie, se confirma tal situación ya que 2 de los 3 accesos protésicos desarrollaron robo en postoperatorio inmediato y antes de los 6 días, el tercero a los 4 meses. Los autólogos lo presentaron 1-2 años después de construido, incluyendo uno a los 6,5 años.

Ninguna evaluación preoperatoria predice el robo, aunque algunas características del paciente y de las técnicas quirúrgicas previas pueden ayudar. Los pacientes en riesgo mayor son los diabéticos por la presencia de enfermedad oclusiva de arterias medianas y pequeñas, también la presencia de múltiples accesos previos, debido a la ubicación más proximal del nuevo acceso y a la pérdida de arterias donantes. Esta situación se confirma, en esta serie, ya que el $100 \%$ de los pacientes tiene acceso en pliegue del codo, varios con múltiples fístulas arteriovenosas previas (2, 3 y 5) y la mayoría eran diabéticos (61\%).

La utilización de estudios no invasivos, sólo son útiles para documentar la mayor probabilidad del riesgo de presentación de esta grave complicación. El uso de la fotopletismografía digital e índices digito/brazo disminuídos en el preoperatorio, sólo pueden sugerir el riesgo de desarrollo tardío de isquemia, no existiendo valores absolutos para este índice, que permitan su predicción. El ecodoppler es útil para evaluar etiología del robo, especialmente la presencia de inversión del flujo arterial, y tamaño del ostium del acceso, y también para controlar la efectividad de la modificación del acceso. Entre los invasivos, la angiografía permite conocer presencia de estenosis en el flujo de entrada o más distal en la arteria donante.

El diagnóstico de robo es clínico ${ }^{3}$, basado en las manifestaciones de isquemia conocidas, que pueden aparecer en este grupo de pacientes, la decisión de intervenir depende de la severidad del cuadro; fue nuestro criterio, sólo completado con pletismografía y ecodoppler.

En la actualidad se dispone de varias alternativas de tratamiento ${ }^{4}$, que incluyen la ligadura del acceso, técnicas de reducción del flujo sanguíneo al acceso (banding, sutura parcial o aplicación de clips) que en general conducen a pérdida del acceso y necesidad de crear uno nuevo. Para aquellos pacientes, cuyo acceso es el último o esencial para mantener la hemodiálisis, la $\mathrm{RDLI}^{5,6}$ es un procedimiento durable y efectivo, ya que alivia la isquemia y mantiene la permeabilidad del acceso.

Los resultados publicados son buenos, con alivio o mejoría completa de la isquemia, mayor al $70 \%$. La permeabilidad del bypass son elevadas; Knox ${ }^{7}$, en una serie de 55 pacientes, reportó cifras de $86 \%$ a 12 meses y $80 \%$ a 48 meses. Korzets ${ }^{8}$, en 11 pacientes, reportó permeabilidad de $90 \%$ y $80 \%$ a 12 y 24 meses. Sessa ${ }^{9}, 94 \%$ a 12 meses en 18 pacientes.

Pese a que el RDLI ha cambiado el tratamiento del robo, no es totalmente efectivo en el alivio de la isquemia, desconociéndose la etiología, proponiéndose como causa la presencia de segmento de menor presión en la arteria humeral vecina a la fístula, descrito por Wixon ${ }^{10}$ y confirmado por Reifsnyder ${ }^{11}$ en una serie de 9 pacientes con robo, observando progresiva mejoría de las presiones sistólicas en la medida que se alejaba de la fístula, mejorando hasta la arteria axilar. Dados estos hallazgos, se debería considerar que el bypass se origine lo más alejado del acceso, sobre los $5 \mathrm{~cm}$ recomendados en la técnica original ${ }^{12}$, sin embargo, en nuestros pacientes, no observamos ninguna diferencia, ya que 3 bypass originados a $10 \mathrm{~cm}$ prosiguieron con dolor y no impidieron la amputación digital; uno originado a $6 \mathrm{~cm}$ persistió con dolor; tal vez el uso de la arteria axilar como donante, podría haber mejorado nuestros resultados. Según Illig ${ }^{13}$, el éxito del RDLI en la mejoría de la perfusión, obedece a presencia de alta presión en el punto en que el flujo se divide para irrigar la mano y la fístula, permitiendo flujo anterógrado por el bypass a una extremidad con menor presión. La resistencia creada por el segmento arterial entre la fístula y el nuevo bypass explicaría esta alta presión. 
La alta mortalidad operatoria (13\%) y a largo plazo (56,5\%), observadas en nuestra serie es concordante con la de $58 \%$, descrita por $\mathrm{Yu}^{14}$, en una serie de 24 pacientes; concluyendo en que los pacientes con robo especialmente diabéticos, representan un grupo de alto riesgo, con expectativas de vida limitadas.

Por lo anterior, pese a efectividad del RDLI, todos los esfuerzos se deben orientar a la prevención de la isquemia, para disminuir la morbimortalidad de esta complicación, evitando en lo posible la confección del acceso en pliegue del codo, uso de flujo aferentes más proximales, limitar el tamaño de la anastomosis y uso de catéteres de hemodiálisis permanentes en aquellos pacientes con comorbilidades múltiples y esperanza de vida limitada.

Los resultados obtenidos, demuestran que esta técnica elimina la isquemia en la mayoría de los pacientes, no comprometiéndose la permeabilidad del acceso, también apoyan la postura del uso de RDLI como procedimiento de elección en la corrección de la isquemia severa de la extremidad después de construcción de un acceso vascular para hemodiálisis.

\section{Referencias}

1. Schanzer H, Schwartz M, Harrington E, Haimov M. Treatment of ischemia due to steal by arteriovenous fistula with distal artery ligation and revascularization. J Vasc Surg 1988;7:770-3.

2. Lazarides M, Staramos D, Kopadis G, Maltezos C, Tzilalis V, Georgiadis G. Onset of arterial steal following proximal angioaccess: inmediate and delayed types. Nephrol Dial Transplant. 2003;18:2387-90.

3. Galleguillos I, Aguiló J, Pizarro H, Vallejos C, Vergara J, Schonffeltd G, y col. Isquemia sintomática en accesos vasculares para hemodiálisis. Rev Chil Cir. 2007;59:348-52.

4. Nash P, Wilson S. Strategies for management of ische- mia steal syndrome. Sem Vasc Surg. 2007;20:184-7.

5. Van der Meer J, Zeebregts C, Tiellin I, Verhoeven E, Van den Dungen J. Modified distal revascularization with interval ligation procedure for steal syndrome after arteriovenous fistula creation for hemodialysis access. Vascular 2007;15:226-30.

6. Walz P, Ladowski J, Hines A. Distal revascularization and interval ligation (DRIL) procedure for the treatment of ischemic steal syndrome after arm arteriovenous fistula. Ann Vasc Surg. 2007;21:468-73.

7. Knox R, Berman S, Hughes J, Gentile A, Mills J. Distal revascularization-interval ligation: a durable and effective treatment for ischemic steal syndrome after hemodialysis access. J Vasc Surg. 2002;36:2.

8. Korzets A, Kantarovsky A, Lehmann J, Sachs D, Gershkovitz R, Hasdan, et al. The DRIL procedure: a neglected way to treat the steal syndrome of the hemodialysed patient. Isr Med Assoc J. 2003;5:782-5.

9. Sessa C, Riehl G, Porcu P. Treatment of hand ischemia following angioaccess surgery using the distal revascularization-interval ligation technique with preservation of vascular access: description of an 18-case series. Ann Vasc Surg. 2004;18:685-94.

10. Wixon C, Hughes J, Mills J. Understanding strategies for the treatment of ischemic steal syndrome. J Am Coll Surg. 2000;191:301-10.

11. Reifsnyder T, Arnaoutakis G. Arterial pressure gradient of upper extremity arteriovenous access steal syndrome: treatment implications. Vasc Endovasc Surg. 2010;44:650-3.

12. Schanzer H, Skladany M, Haimov M. Treatment of angioaccess induced ischemia by revascularization. J Vasc Surg. 1992;16:861-6.

13. Illig K, Surowiec S, Shortell C, Davies M, Rhodes J, Green R. Hemodynamics of distal revascularizationinterval ligation. Ann Vasc Surg. 2005;19:199-207.

14. Yu S, Cook P, Canty T, Mc Ginn F, Taft P, Hye R. Hemodialysis-related steal syndrome: predictive factors and response to treatment with the distal revascularization-interval ligation procedure. Ann Vasc Surg. 2008;22:210-4. 